\title{
НОВЫЙ КОНТРОЛЛЕР ОМRON SYSMAC С ФУНКЦИЯМИ ИСКУССТВЕННОГО ИНТЕЛЛЕКТА
}

\author{
Алексей БЛУДОВ, Павел ШЕШЕРА
}

Компания Omron выпустила новый контроллер Sysmac с функциями машинного обучения и искусственного интеллекта. Это решение позволит промышленным предприятиям поднять эффективность технологического процесса на новый уровень. В статье описаны инновационные особенности данного решения и его преимущества перед привычными «облачными» технологиями.

Технологии искусственного интеллекта (ИИ) и машинного обучения сегодня все шире используются для выполнения таких трудоемких прикладных задач, как автоматизация (роботизация) перевода, информационный поиск, распознавание образов и речи, анализ данных и пр. В настоящее время эти технологии внедряются на производстве, транспорте и в сфере потребительских услуг, позволяя любому предприятию добиться ощутимых конкурентных преимуществ.

В автоматизации промышленности ИИ становится востребованным как одно из возможных средств решения задачи повышения эффективности в рамках цифровой трансформации производственных предприятий.

Большинство разработчиков систем искусственного интеллекта и машинного обучения для промышленных предприятий создают «облачные» решения. В такой системе производственные данные собираются с технологических линий, передаются в ИТ-систему и «облако», где обрабатываются специальным программным обеспечением в целях оптимизации производственного процесса. Компания Omron Electronics, являясь мировым лидером в производстве электронных компонентов промышленной автоматизации, предлагает оригинальное решение на основе технологии искусственного интеллекта для применения на производстве.

Это решение берет начало в новой концепции Omron под названием i-Automation («Инновационная автоматизация»). В рамках данной концепции сформулированы три ключевые задачи, решение которых позволит значительно повы- сить конкурентоспособность промышленного предприятия:

$\rightarrow$ обеспечение устойчивости производства;

$\rightarrow$ повышение эффективности процессов;

$\rightarrow$ защита бренда.

Важную роль в решении этих задач играет увеличение общей эффективности оборудования (OEE), на которую непосредственно влияют время внеплановых простоев и доля брака в выпускаемой продукции. Для сокращения простоев и затрат на ремонты, а также повышения качества выпускаемой продукции на передовых производственных предприятиях внедряются системы предиктивного (прогностического) обслуживания.

Именно для реализации задач предиктивного обслуживания, оптимизации работы оборудования и улучшения качества продукции Omron оснастил свои новые модели универсальных машинных контролеров семейства Sysmac (рис. 1) функциями искуственного интеллекта и машинного обучения (Sysmac ИИ). Уникальность решения заключается в том, что искусственный интеллект реализован на уровне АСУ ТП и как дополнительная функция, поскольку основная задача контроллера - управление производственным процессом - не изменилась.

При этом синхронно с выполнением задач управления контроллер Sysmac с искусственным интеллектом в режиме реального времени (с периодом 125 или 500 мкс) собирает производственные данные, выделенные как значимые («признаки»), и анализирует их согласно заложенной в него модели, то есть выполняет так называемые «граничные вычисления» и обработку данных. При выявлении отклонений в работе оборудования, которые искусственный интеллект интерпретирует как поломку либо угрозу ее возникновения, контроллер мгновенно корректирует управляющие воздействия на исполнительные механизмы, что позволяет свести к минимуму негативные последствия и предотвратить выпуск продукции ненадлежащего качества. Отсюда следует главное преимущество нового решения Omron перед 


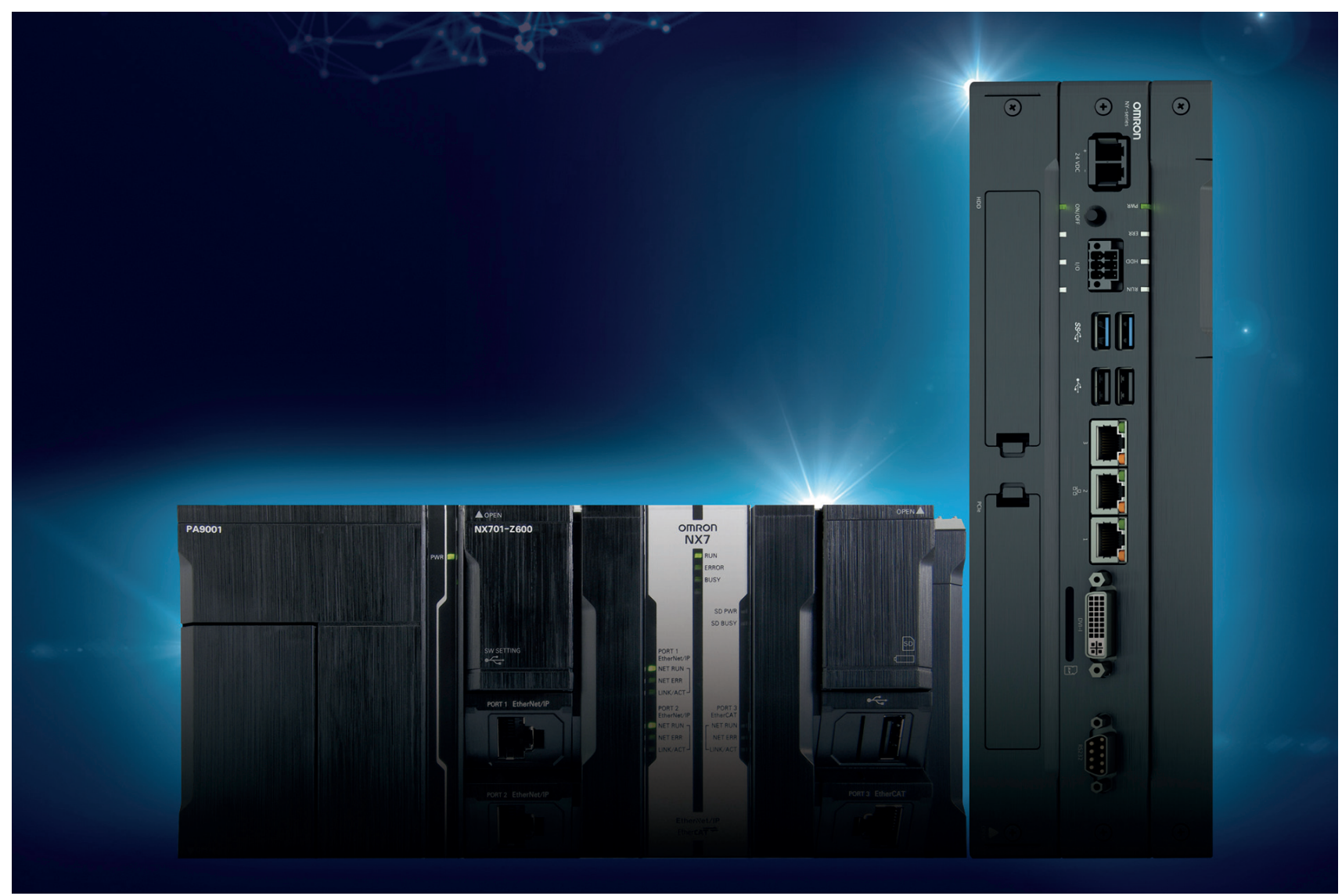

Puc. 1. Контроллеры Sysmac с искусственным интеллектом

стандартными «облачными» решениями: АСУ ТП на базе контроллера Sysmac с искусственным интеллектом способна не только информировать производственный персонал о появлении отклонений в работе производственного оборудования, но и оперативно (проактивно) на них реагировать.

Если же система Sysmac ИИ регистрирует неисправности в работе оборудования, которые не превышают критических значений, приводящих к поломкам и остановкам, то в полной мере реализуется концепция предиктивного обслуживания: производственный персонал получает информацию о возникновении дефекта в конкретном узле установки уже на начальном этапе (например, о дефекте в результате износа) и имеет возможность заранее назначить его ремонт или замену на следующую плановую остановку. Иными словами, обслуживание оборудования выполняется без потери производительности и не приводит к нарушению производственного плана. Таким образом, внедрение системы Sysmac ИИ позволяет предприятию уйти от неэффективной системы планово-предупредительных ремонтов. При этом требования к квалификации и опыту обслуживающего персонала существенно снижаются.

у системы Sysmac ИИ есть еще одно важное преимущество: она не требует подключения к локальной сети или Интернету для передачи данных и их обработки на верхнем уровне, поэтому не приходится создавать дорогую и сложную ИТ-инфраструктуру. Помимо ощутимой экономии средств, такой подход существенно повышает безопасность и сохранность данных - вся информация гарантированно остается внутри производственной площадки предприятия, даже при интеграции АСУ ТП с информационной системой компании.

Поскольку работа искусственного интеллекта требует мощных ресурсов, аппаратная составляющая системы Sysmac ИИ включает самые производительные машинные контроллеры Sysmac Omron серий NX7 и NY5, оснащенные многоядерными/многопоточными микропроцессорами Intel Core i7. Традиционные ПЛК с их элементной базой не способны осуществлять функции управления и обработки данных ИИ параллельно в режиме реального времени.

Чтобы дать представление о возможностях искусственного интеллекта новых контроллеров Sysmac, перечислим некоторые из их характеристик: до 2048 переменных для анализа, детектирование до 128 событий оборудования, до 16 признаков для каждого события оборудования.

Программное обеспечение Omron для искусственного интеллекта реализует функции машин- 
ного обучения на базе алгоритма Isolation Forest и используется на этапах настройки и отладки системы. Машинное обучение происходит следующим образом: в нормальном режиме работы с производственной линии собираются данные, из общего массива данных о состоянии оборудования выделяется только та информация, которая является значимой для диагностики неисправностей, так называемые признаки. На основе анализа входных сигналов строится модель, которая загружается в контроллер. Модель способна различать несколько режимов работы производственного оборудования.

Другая разновидность ПО для разработчиков систем на базе аппаратно-программного комплекca Sysmac ИИ представляет собой готовую библиотеку функциональных блоков для контроллера. С помощью данного ПО реализуются диагностика и предиктивное обслуживание трех типовых механизмов производственного оборудования: пневмоцилиндра, шарико-винтовой передачи, а также ременной передачи со шкивом. Диагностируются, например, такие типовые неисправности, как износ резиновых прокладок цилиндра, ослабление ремня ременной передачи, износ ШВП и т. п.

Готовые программные компоненты не требуют предварительного анализа данных и проработки входных сигналов для выявления предиктивных признаков. Это ускоряет разработку и внедрение системы на базе Sysmac ИИ для указанных типов узлов технологических установок. Важно упомянуть, что Omron планирует расширять свою библио-

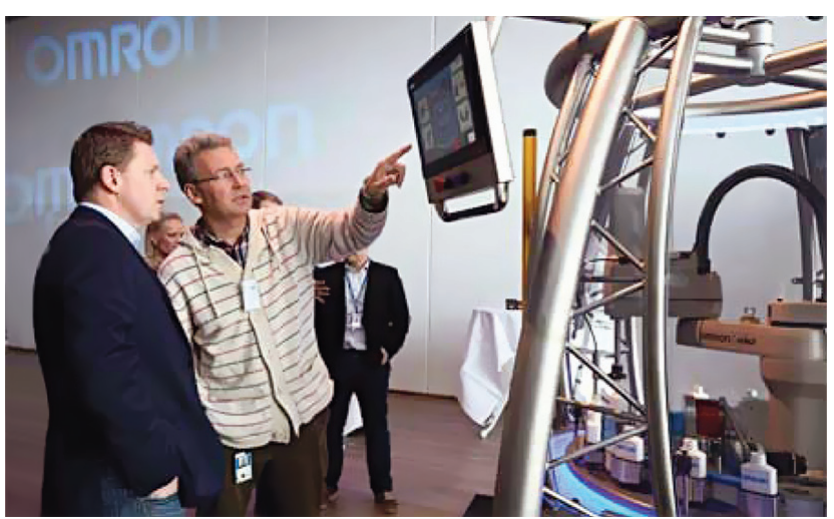

Puc. 2. Роботизированная установка непрерывного розлива на базе нового контроллера Sysmac c искусственным интеллектом теку готовых функциональных блоков предиктивного обслуживания для самых ходовых узлов производственного оборудования.

Компания Omron только начинает выводить свое новое решение на рынок, поэтому планируется, что на начальном этапе машинное обучение и создание моделей искусственного интеллекта для заказчика будут выполнять специалисты компании. При таком подходе заказчики получат отлаженную и готовую к эксплуатации систему с функциями предиктивного обслуживания. Одновременно с этим в целях наилучшего результата предполагается тесно сотрудничать с технологическим персоналом, обслуживающим модернизируемое или устанавливаемое производственное оборудование.

На Ганноверской промышленной ярмарке, которая проходила 1-5 апреля 2019 года, компания Omron представила первый в мире стенд, демонстрирующий преимущества искусственного интеллекта реального времени в технологическом процессе (рис. 2). SCARA-робот Omron выполняет подачу дозирующего устройства синхронно с движением тары. Если внешние признаки (информация с энкодера подающего стола, датчиков вибрации и температуры; степень загрязненности оптического датчика наличия тары; данные по скорости и моменту сервомоторов и временные показатели) согласно заложенной в память контроллера модели не сигнализируют о существенных отклонениях, то носик дозирующего устройства опускается внутрь тары, емкость заполняется и передвигается дальше. Однако в случае обнаружения неполадок выдвижения дозатора не происходит, а установка избегает вынужденного останова вследствие механических повреждений конструкций и розлива продукта.

В рамках концепции i-Automation компания Omron разработала передовую продукцию с искусственным интеллектом. Ее внедрение будет способствовать ускорению цифровой трансформации производства и созданию высокоэффективных предприятий Четвертой промышленной революции.

\section{Блудов Алексей Владимирович - менеджер по продукции «Автоматизация», Омрон Электроникс}

\section{ШЕШЕРА Павел Сергеевич -}

инженер по применениям, Омрон Электроникс

000 «мрон Электроникс» сайт: industrial.omron.ru 


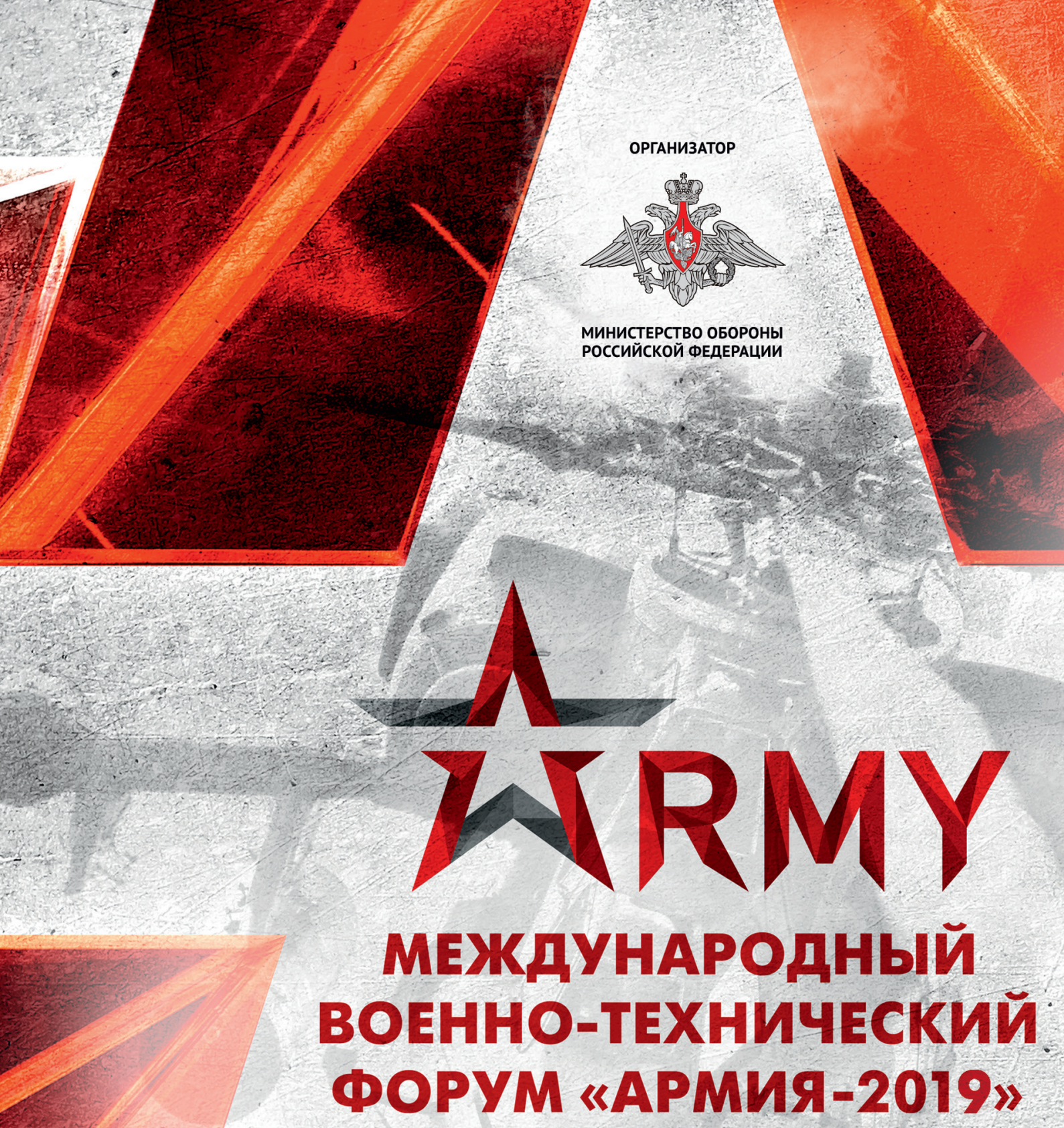

\section{5-30 Июня ПАТРИОТ ЭКСПО}

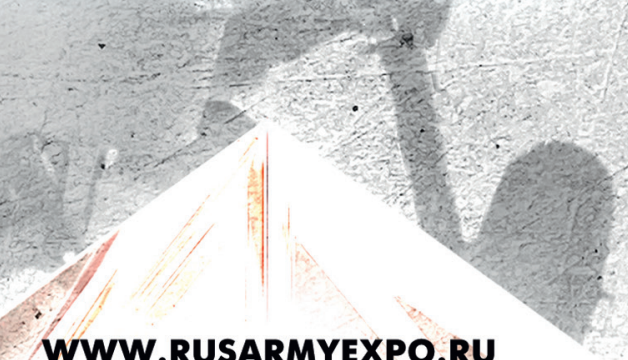

WWW.RUSARMYEXPO.RU

ВЫСТАВОЧНЫЙ ОПЕРАТОР

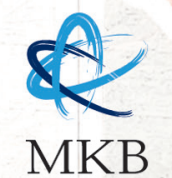

